\title{
2.2 Wohnraum: barrierefrei oder barrierereduziert?
}

Im für diese Arbeit relevanten wohnungspolitischen Teilbereich finden sich verschiedenste Begrifflichkeiten, um den entsprechend ausgestatteten Wohnraum zu beschreiben. »Altersgerecht « oder »behindertengerecht « sind dabei keinesfalls einheitlich definiert: »Eine gesetzliche bzw. allgemeingültige Definition [...], in der die Anforderungen beschrieben und verbindlich gemacht werden, gibt es nicht. Dies führt in der Praxis zu sehr unterschiedlichen Handhabungen « (BMVBS 2011: 25). Mit den verwendeten Begriffen gehen entweder klare Verbindlichkeiten einher - barrierefrei nach DIN 18040-2 -, oder aber es erfolgt lediglich eine Schlagwortsetzung in Richtung der »Kund*innen«-Mieter*innen, selbstnutzende Eigentümer*innen - durch rechtlich undefinierte Begriffe.

Zur Erhellung dieses begrifflichen Dschungels erläutert dieses UnterKapitel die diese Forschungsarbeit leitenden Begriffe. Dies geschieht zunächst über die normative UN-Behindertenrechtskonvention und das Behindertengleichstellungsgesetz. Es wird dann weitergeführt mit der Barrierefreiheit nach der technischen Norm 18040-2 vom Deutschen Institut für Normung e.V. (DIN). Daraufhin wird die Barrierereduzierung nach den Technischen Mindestanforderungen der Kreditanstalt für Wiederaufbau (KfW) eingeführt. Auf die Nutzung von Begriffen wie »seniorengerecht« oder »behindertengerecht « wird verzichtet, da keinerlei Konsens hinsichtlich deren inhaltlicher Ausgestaltung besteht.

UN-Behindertenrechtskonvention und Behindertengleichstellungsgesetz

Für den Gedanken der Barrierefreiheit ist die UN-Behindertenrechtskonvention (UNBRK) wegweisend. Ihre Prinzipien der Autonomie und Inklusion wurden von Deutschland im Jahr 2009 ratifiziert und sind damit rechtlich bindend. Im Vergleich zum früheren Verständnis einer »Behinderung « findet eine Akzentverschiebung statt: »Man ist nicht behindert, sondern man wird behindert « (DIMR 2019: 11). Damit ist festgeschrieben, dass Menschen durch unzureichende Hilfsmittel und Umgebungen »behindert werden«, anstatt pauschal »behindert zu sein« (ebenda, vgl. auch BMAS 2016b: 115).

Der Begriff »Barrierefreiheit« selbst ist in der UN-BRK nicht explizit genannt, vielmehr werden verschiedene normative Leitlinien definiert. So bedeutet nach Artikel 2 eine Diskriminierung aufgrund von Behinderung »jede Unterscheidung, Ausschließung oder Beschränkung aufgrund von Behinderung, die zum Ziel oder zur Folge hat, dass das auf die Gleichberechtigung mit anderen gegründete Anerkennen, Genießen oder Ausüben aller Menschenrechte und Grundfreiheiten [...] beeinträchtigt oder vereitelt wird « (Behindertenbeauftragter 2017: 8). ${ }^{2}$ Mit einer Nichtdiskriminierung wiederum sind verschiedene Arten der $\mathrm{Zu}$ gänglichkeit verbunden, wie Artikel 9 (1) mit dem Verweis auf einen gleichberechtigten Zugang zur physischen Umwelt und die dadurch ermöglichte eigenständige Lebensführung konkretisiert (ebenda: 13). Und schließlich erkennt Artikel 19 Menschen mit Behinderung die "gleichen Wahlmöglichkeiten« wie anderen Menschen zu, etwa dadurch, dass sie entscheiden können, wo und mit wem sie leben (ebenda: 17f.).

2 Gleichzeitig heißt es aber auch: »[...] bedeutet >angemessene Vorkehrungen notwendige und geeignete Änderungen und Anpassungen, die keine unverhältnismäßige oder unbillige Belastung darstellen [...] « (Behindertenbeauftragter 2017: 8). 
Neben der UN-Behindertenrechtskonvention stellen das Behindertengleichstellungsgesetz (BGG) des Bundes und darauf aufbauend die Gesetze der Länder eine zentrale normative Leitlinie dar. Hier definiert $\$ 4$ : »Barrierefrei sind bauliche und sonstige Anlagen, Verkehrsmittel, [...], wenn sie für behinderte Menschen in der allgemein üblichen Weise, ohne besondere Erschwernis und grundsätzlich ohne fremde Hilfe zugänglich und nutzbar sind« (Bundesgesetzblatt 2002: 1468). Dieses Verständnis ähnelt der UN-BRK, nach der Menschen nicht pauschal behindert sind, sondern durch unangemessene Umgebungen behindert werden. Das Bundes-BGG wurde erstmals 2002 erlassen und unterliegt seitdem steten Wandlungsprozessen (DIMR 2019: 22).

Sowohl bei der UN-BRK als auch beim BGG handelt es sich um sogenannte Definitionsnormen, die einem Begriff einen normativen Inhalt zuschreiben. Daraus ergeben sich noch keine konkreten Pflichten - vielmehr sind diese Normen »bei der Auslegung von Vorschriften heranzuziehen, die solche Rechte oder Pflichten enthalten « (Bundesfachstelle Barrierefreiheit 2020). So zählt auch das Bauordnungsrecht zu solchen Vorschriften, die sich auf Artikel 4 BGG berufen und eine konkrete empirische Anwendbarkeit gewährleisten (vgl. Kapitel 6.1). Diese empirische Anwendbarkeit liefert im Folgenden die DIN 18040-2. Mit ihr wird ein Perspektivwechsel vom normativen hin zum empirischmessbaren Verständnis von baulicher Barrierefreiheit vorgenommen.

DIN-Norm 18040-2: Barrierefreiheit

Für das barrierefreie Bauen ist die bautechnische Normenreihe DIN 18040 handlungsleitend. Sie besteht aus drei Teilen: Während DIN 18040-1 für öffentlich zugängliche Gebäude gilt und DIN 18040-3 für den öffentlichen Verkehrs- und Freiraum, ist für den privaten Wohnraum der Normenteil 2 zuständig: Er formuliert Voraussetzungen zu Wohngebäuden und ihren Außenanlagen, den Wohnungszugängen und der inneren Wohnungsausstattung, nach denen ein Gebäude barrierefrei ist (WM Baden-Württemberg 2017: 10f.; DIN 2020).

Dem Vorwort von DIN 18040-2 folgend berücksichtigt sie die Bedürfnisse von Menschen mit unterschiedlichsten körperlichen, kognitiven oder motorischen Einschränkungen (bspw. Menschen mit Seh- und Hörbehinderung, groß- und kleinwüchsige Menschen, ältere Menschen) (DIN 2020). Dass eine pauschale Norm nicht allen individuellen Bedürfnissen eines jeden/einer jeden Einzelnen gerecht werden kann, ist dabei anerkannt, weshalb von einer Art Mindeststandard der baulichen Barrierefreiheit gesprochen wird (VDI 2017: 5). 
Tabelle 3: DIN 18040-2: Inhaltsverzeichnis (nach HMWEVW 2013: 33; FM RLP/MSAGD 2017)

\begin{tabular}{|c|c|}
\hline \multicolumn{2}{|l|}{ Vorwort } \\
\hline $\begin{array}{l}\text { Kapitel 1: } \\
\text { Anwendungsbereich }\end{array}$ & $\begin{array}{l}\text { Unterscheidung in barrierefrei nutzbare Wohnungen und barrierefrei und } \\
\text { uneingeschränkt mit dem Rollstuhl nutzbare Wohnungen }\end{array}$ \\
\hline $\begin{array}{l}\text { Kapitel 2: Normative } \\
\text { Verweisungen }\end{array}$ & Verweise auf weitere relevante Normen \\
\hline Kapitel 3: Begriffe & $\begin{array}{l}\text { 3.1 Bedienelement } \\
\text { 3.2 Bewegungsfläche } \\
\text { 3.3 Blindheit } \\
{[\ldots]}\end{array}$ \\
\hline Kapitel 4: Infrastruktur & $\begin{array}{l}\text { 4.1 Allgemeines } \\
\text { 4.2 Äußere Erschließung auf dem Grundstück } \\
\text { 4.3 Innere Erschließung des Gebäudes } \\
\text { 4.4 Warnen/Orientieren/Informieren/Leiten } \\
\text { 4.5 Bedienelemente, Kommunikationsanlagen, } \\
\quad \text { Ausstattungselemente }\end{array}$ \\
\hline $\begin{array}{l}\text { Kapitel 5: Räume } \\
\text { in Wohnungen }\end{array}$ & $\begin{array}{l}\text { 5.1 Allgemeines } \\
\text { 5.2 Flure innerhalb von Wohnungen } \\
\text { 5.3 Türen, Fenster } \\
\text { 5.4 Wohn-, Schlafräume und Küchen } \\
\text { 5.5 Sanitärräume } \\
\text { 5.6 Freisitz }\end{array}$ \\
\hline
\end{tabular}

Die DIN 18040-2 setzt sich aus fünf inhaltlichen Kapiteln zuzüglich Vorwort zusammen (vgl. Tabelle 3). Wichtig für die Zugänglichkeit zur Wohnung und die Ausstattung innerhalb der Wohnung sind insbesondere die Regelungen in den Kapiteln 4 und 5. Zentral ist außerdem die Unterscheidung in barrierefreie und eingeschränkt bzw. uneingeschränkt mit dem Rollstuhl nutzbare Wohnungen. Bei der Infrastruktur, also beim Zugang und den sogenannten Erschließungsbereichen - ab Grundstücksgrenze inklusive Hausfluren und Treppenhäusern -, muss eine uneingeschränkte Nutzbarkeit mit dem Rollstuhl gewährleistet sein. Das entspricht unter anderem Bewegungsflächen von 1,50 Metern mal 1,50 Metern und lichten Türdurchgangsbreiten von 90 Zentimetern (VDI 2017: 5; Fachportal nullbarriere.de 2018). Dahingegen wird innerhalb der Wohnung zwischen zwei unterschiedlichen Ausstattungsqualitäten unterschieden. Der RStandard - barrierefrei und uneingeschränkt mit dem Rollstuhl nutzbar - entspricht den Vorgaben für die Infrastruktur, wohingegen beim B-Standard - barrierefrei und eingeschränkt mit dem Rollstuhl nutzbar - bspw. kleinere Bewegungsflächen (1,20 m x $1,20 \mathrm{~m}$ ) und eine lichte Türdurchgangsbreite von 80 Zentimetern gelten. Die Wohnungen nach B-Standard sind für Menschen geplant, welche zwar nicht auf einen Rollstuhl angewiesen sind, aber sonstige Gehhilfen benötigen oder durch eine Sehbehinderung einen veränderten Wohnraumbedarf haben (VDI 2017: 5f.). Der B-Standard stellt damit einen Mindeststandard dar, auf dem der R-Standard aufbaut (vgl. Abbildung 2).

Die DIN-Landschaft unterliegt einem steten Wandel, um jeweils neue technologische, aber auch gesellschaftliche Entwicklungen abbilden zu können. So besteht die Normenreihe 18040 seit dem Jahr 2011 und ersetzt die zuvor gültige DIN 18025 aus 
Abbildung 2: B- und R-Standard nach DIN 1840-2 (eigene Darstellung)

Barrierefrei und eingeschränkt mit dem Rollstuhl nutzbare Wohnungen ("B-Standard")

Barrierefrei und uneingeschränkt mit dem Rollstuhl nutzbare Wohnungen ("R-Standard")

dem Jahr 1992. Im Rahmen einer grundlegenden Überarbeitung wurden erstmals Anforderungen an die Sensorik integriert und sogenannte Schutzziele definiert (DIN 2020; HMWEVW 2013: 3). Schutzziele geben ein Ziel vor, ohne den konkreten Weg zur Erreichung dieses Ziels verbindlich vorzuschreiben: „Die mit den Anforderungen nach dieser Norm verfolgten Schutzziele können auch auf andere Weise als in der Norm festgelegt erfüllt werden« (Rau 2012: 28). Sie gewähren der Bauplanung mehr Flexibilität (Jocher et al. 2016: 22; WM Baden-Württemberg 2017: 113). Zudem arbeitet die Europäische Union an einer DIN EN 17210 »Barrierefreiheit und Nutzbarkeit der gebauten Umgebungen«. Ein erster Entwurf wurde im Mai 2019 veröffentlicht und hätte mit einer Verabschiedung auch Auswirkungen auf die nationalstaatlichen Regelungen der EU-Mitgliedstaaten (bfb barrierefrei bauen 2019b; Fachportal nullbarriere.de 2019). Im Folgenden beziehen sich die Inhalte aber auf die im Jahr 2019 gültige DIN 18040-2.

Wichtig ist, dass DIN-Normen als technische Regeln noch keine automatische Gültigkeit besitzen. Das Deutsche Institut für Normung e.V. selbst ist ein eingetragener privatwirtschaftlicher Verein, der nach seiner Satzung zunächst dazu dient, »zum Nutzen der Allgemeinheit [...] in geordneten und transparenten Verfahren die Normung und Standardisierung anzuregen, zu organisieren, zu steuern und zu moderieren « (DIN 2015: 3). Erst mit der Übernahme in die Landesbauordnungen bzw. in die dahinterliegenden Technischen Baubestimmungen werden ihre Inhalte verbindlich. Inwieweit die 16 Bundesländer dabei die komplette DIN 18040-2 übernehmen oder nur Teile von ihr, ist unterschiedlich (vgl. Kapitel 6.1.3). Zudem wurde die DIN 18040-2 für Neubauvorhaben mit dem Grundsatz konzipiert, diese Anforderungen bei Umbauten im Bestand »sinngemäß anzuwenden« (Bayerische Architektenkammer et al. 2014: 19). Gerhard Loeschke - Obmann im DIN-Ausschuss für den barrierefreien Neubau und maßgeblich bei der Entwicklung der KfW-Technischen Mindestanforderungen beteiligt - erläutert dazu:

»Diese nach DIN ausgerichtete Barrierefreiheit zeigt sich [...] im Wohnungsbestand der frühen Nachkriegsbauten wenig zielführend und zwar sowohl im monetären als auch im nutzungsspezifischen Sinne. Das Festhalten an >gänzlich < barrierefreien Standards würde insbesondere im Wohnungsbestand eine gravierende Investitionslücke heraufbeschwören« (Loeschke/Pourat 2009: 7, vgl. auch KfW 0.J.).

Technische Mindestanforderungen der KfW: Barrierereduzierung Loeschke/Pourat (2009) verweisen damit auf eine weitere begriffliche Kontroverse: Auch wenn normative Leitlinien eine umfassende Barrierefreiheit vorsehen und die DIN 18040-2 versucht, diese Barrierefreiheit für den Neubau zu operationalisieren, so las- 
sen sich ihre Kriterien teils schwierig auf Bestandsbauten übertragen. Selbst wenn theoretisch ab morgen alle Wohngebäude »barrierefrei und uneingeschränkt mit dem Rollstuhl nutzbar« gebaut würden, sieht sich der deutsche Wohnungsmarkt einem immensen Wohnungsbestand mit vielen Barrieren gegenüber.

Hier gibt es verschiedene Anstrengungen, auch für die Bestandsmodernisierung entsprechende Prüfsteine zu formulieren. Der Kriterienkatalog der Förderbank KfW für das Förderprogramm »Altersgerecht Umbauen« ist dabei der bekannteste. Die KfW ist eine nationale Förderbank in der Rechtsform einer Anstalt des öffentlichen Rechts. Bereits seit ihrer Gründung im Jahr 1948 fördert sie Investitionen im In- und Ausland, unter anderem in der Wohnbauförderung. Durch ihre Finanzkraft übt sie auch auf die Landesförderbanken eine große Orientierungswirkung aus (KfW 2020b; KfW 2020a, vgl. ausführlich Kapitel 6.2.2). Zum Jahr 2019 bestanden im Programm »Altersgerecht Umbauen« sieben Bereiche zur Förderung von barrierereduzierenden Umbaumaßnahmen (KfW 2019a: 2ff.):

a) Wege zum Gebäude und Wohnumfeldmaßnahmen

b) Eingangsbereich und Wohnungszugang

c) Vertikale Erschließung/Überwindung von Treppen und Stufen

d) Anpassung der Raumgeometrie/Raumzuschnitt und Schwellenabbau

e) Badumbau/Maßnahmen an Sanitärräumen

f) Orientierung, Kommunikation und Unterstützung im Alltag

g) Gemeinschaftsräume und Mehrgenerationenwohnen

Die KfW hat zusätzlich den Standard »Altersgerechtes Haus« für den Fall entwickelt, dass Maßnahmen in allen sieben Förderbereichen realisiert werden (KfW 2019a: 10). Angesichts der bestehenden terminologischen Kontroversen verstärkt dieser Standard wiederum die begriffliche Unschärfe im Feld, da zusätzlich zur Barrierefreiheit nach DIN 18040-2 und der Barrierereduzierung nach KfW-Technischen Mindestanforderungen das »Altersgerechte Haus« eingeführt wird.

An dieser Stelle soll der Verweis auf diese Mindestanforderungen genügen. Ebenso wie bei der DIN 18040-2 werden für alle sieben Förderbereiche Kriterien definiert - allerdings nach eigener Aussage technisch realisierbar und ökonomisch finanzierbar für den Bestandsumbau (vgl. KfW 2019a und Kapitel 6.2.2). Es bleibt festzuhalten: Die Begriffe »barrierereduziert « und »altersgerecht « sind nicht geschützt und dürfen entsprechend auch verwendet werden, wenn die KfW-Kriterien nicht eingehalten sind. Dennoch ist es in dieser Forschungsarbeit wichtig, auch bei Bestandsumbauten und damit einhergehenden »Reduzierungen « von Barrieren sprechfähig zu sein. Verschiedene Förderprogramme und Beratungsangebote adressieren den Bestandsumbau und erfüllen häufig im Ergebnis nicht die in der DIN 18040-2 formulierten Kennzahlen zur Barrierefreiheit. Somit wurde sich hier für den Begriff des barrierereduzierten Wohnraums entschieden. Die entsprechend umgebauten Wohnungen oder Wohnumfelder können eine signifikante Wohnverbesserung für die Nutzer*innen bedeuten, auch wenn sie weniger strukturiert fassbar sind. »Barrierereduziert « wird in dieser Arbeit deshalb in 
Anlehnung an die Technischen Mindestanforderungen der KfW verstanden, aber nicht ausschließlich auf diese bezogen.

Fazit zu den Begriffen Barrierefreiheit und -reduzierung

Zum barrierefreien bzw. -reduzierten Wohnraum kursieren zahlreiche Formulierungen, von denen die meisten nicht definiert oder geschützt sind. Die hier eingeführten Begriffe

- Barrierefreiheit nach Behindertengleichstellungsgesetz (BGG),

- Barrierefreiheit nach DIN 18040-2 (R- und B-Standard) und

- Barrierereduzierung nach den Technischen Mindestanforderungen der KfW

haben dabei alle ihre jeweilige Berechtigung: Während das BGG die normativen Leitlinien setzt, operationalisiert die DIN 18040-2 spezifische Kriterien für den barrierefreien Neubau. Die KfW wiederum versucht, diese Kriterien in einem Kompromiss zwischen normativen Zielen, technischer Machbarkeit und Finanzierbarkeit auf den Gebäudebestand anzuwenden. Diese Verständnisse widersprechen sich nicht, sondern setzen je eigene Schwerpunkte. Sie nehmen entweder eine spezifische, praktische Perspektive (DIN 18040-2, Technische Mindestanforderungen der KfW) oder eine abstraktere, normative Perspektive (BBG) ein. Diese Forschungsarbeit spricht aufgrund der Nähe zu den in der Politik genutzten Verständnissen von Barrierefreiheit nach der DIN 18040-2 sowie von Barrierereduzierung nach den Technischen Mindestanforderungen der KfW. Die UN-Behindertenrechtskonvention und die Behindertengleichstellungsgesetze der Länder prägen die politischen Prozesse durch ihre normativen Zielsetzungen aber durchaus und sind im weiteren Verlauf unter den institutionellen Rahmenbedingungen zu diskutieren (vgl. Kapitel 4.5.1).

Abschließend sei noch ein letzter Hinweis zu einem mit der Barrierefreiheit verbundenen juristischen Dilemma angeführt, welches Welti (2013: 26ff.) am abgesenkten Bordstein verdeutlicht: Während dieser für die Rollstuhlfahrerin »Barrierefreiheit« bedeute, gefährde er eine sehbehinderte Person aufgrund der fehlenden Orientierungsfähigkeit. Daraus ergibt sich der Spagat, zwischen individuellen Bedürfnissen und einer generalisierten Anforderung für eine unbestimmte Menge an Menschen mit unterschiedlichen Beeinträchtigungen abwägen zu müssen. Erschwerend dazu kommt die Unterscheidung in sogenannte Tatsachenfragen und normative Rechtsfragen: „Ob ein Rollstuhlfahrer Zugang zum Gebäude auch durch den Hintereingang bekommt, ist eine Tatsachenfrage. Ob es sich dabei noch um die allgemein übliche Weise ohne besondere Erschwernis handelt, ist eine normative Rechtsfrage (Welti 2013: 29). Diese mit den jeweiligen Begriffen verbundenen Kontroversen werden in den Fallstudien (vgl. Kapitel 7) aufgearbeitet: Was in der DIN 18040-2 enthalten ist und was dann tatsächlich in das Bauordnungsrecht überführt wird, ist Bestandteil politischer Prozesse, deren Grundlage auch normative und symbolische Debatten sind. 
